\title{
6. SCHLUSSBETRACHTUNG
}

Als in der Forschung noch von der Abhängigkeit des Avignonesischen Papsttums vom König von Frankreich gesprochen wurde, erkannte C. Höfler 1871, $\mathrm{da} B$ »diese zwölf Lustren, ausgefüllt mit dem Pontificate von sechs Päpsten und selbst gipfelnd in der glanzvollen Regierung des dritten derselben, des Limousiners Clemens VI., nicht [...] ein Zustand der unfreiwilligen Sklaverei und der Knechtschaft der Päpste, sondern weit mehr ihres größten Absolutismus waren ${ }^{1}$. Daß diese Erkenntnis rund 100 Jahre benötigte, um sich durchzusetzen, wirft ein bezeichnendes Licht auf die vermeintliche Objektivität von Historiographie. Der von Birgitta von Schweden als harsche Aufforderung formulierten Bemerkung Flectat cardinales ad velle suum ${ }^{2}$ war Clemens VI. im Laufe seines Pontifikates nachgekommen. Beharrlich hatte er die Ergänzung des Kardinalskollegs betrieben und dabei erste Erfolge erzielt. Fast die Hälfte der Neukreierten stand in enger verwandtschaftlicher Bindung zu ihm, und von den übrigen war fundamentale Kritik an seiner Amtsführung nicht zu erwarten. Dies galt auch für die durch Intervention Frankreichs dem Papst anempfohlenen Persönlichkeiten ${ }^{3}$.

Auskunft über die Vorstellungen Clemens' VI. vom Kardinalat geben seine anläßlich der Kreationen und der Rückkehr von Kardinallegaten gehaltenen Collationes. In ihnen finden sich bemerkenswerte Aussagen: Aufgeworfen wird die Frage, über welche Qualifikationen ein guter Kardinal bzw. Legat verfügen müsse, um nutzbringend zum Wohl des Papstes und der Kirche agieren zu können. Artikuliert werden also Idealvorstellungen, deren Aktualisierung im hic et nunc in einem zweiten Schritt ebenfalls Behandlung findet. In einem dritten Schritt erfolgt die Rückbindung der solcherart realisierten Eigenschaften an die Person des Papstes. Insbesondere im Fall der Legationscollationes wird deutlich, daß Clemens VI. seine Kardinallegaten nur selten kritisierte, daß er sich vielmehr eines rhetorischen Vorgehens bediente, das auf Nennung von Kritik verzichtete und durch inhaltliche Leerstellen ersetzte: Wichtig war damit nicht nur das, was gesagt, sondern auch das, was verschwiegen bzw. worauf durch versteckte Andeutungen hingewiesen wurde ${ }^{4}$. So kann

1 Constantin HöflER, Die avignonesischen Päpste, ihre Machtfülle und ihr Untergang, in: Almanach der kaiserlichen Akademie der Wissenschaften 29 (1871) S. 231-285, hier S. 250.

${ }^{2}$ BiRGITTA von Schweden, Revelaciones, hg. v. Hans AILI, Stockholm 1992, lib. 4, c. 49, S. 166.

$3 \mathrm{Vgl}$. Collationes Assumpsi michi duas virgas (Pierre Bertrand), Loquere tu et audiemus (Pierre de Cros), Kap. 4.3., 4.4.

${ }_{4}$ Der Vergleich zwischen den innerhalb der Collationes und der kurialen Korrespondenz getätigten Aussagen erwies sich als conditio sine qua non für die Entschlüsselung einiger Passagen der Collationes. In diesem Zusammenhang sei hier nur auf die Habgier des Guillaume Court hingewiesen, vgl. Kap. 5.2.1.1. Auch bei den von Clemens VI. in Hinblick auf seine Legaten herangezogenen Vergleichsgestalten wie David wurde auf die Nennung charakterlicher Defizite explizit zwar verzichtet, implizit wurden diese Gravamina vom Papst jedoch als bekannt vorausgesetzt, vgl. Collatio Egrediebatur et intrabat, Kap. 5.2.2.2. 
auch scheinbar positiv konnotierten Collationes ein kritischer Subtext unterlegt $\operatorname{sein}^{5}$. Direkte Hinweise auf einzelne kardinalizische Persönlichkeiten sind selten und finden sich zumeist in den humoristisch gefärbten Schlußpassagen, durch die der hochoffiziellen, den Vorgaben des päpstlichen Zeremoniells gehorchenden Redesituation etwas von ihrer Strenge genommen wird ${ }^{6}$. Aussagen über das, was die Kardinäle tatsächlich dachten oder empfanden, finden sich hier jedoch genausowenig wie in den anderen Quellengattungen ${ }^{7}$.

Das Petrusamt erscheint in den Collationes ekklesiologisch korrekt als Gipfelpunkt der Hierarchie, als Amt, das Rechte und Vollmachten umfaßt, die diejenigen anderer Würdenträger übersteigen und unter dem Begriff plenitudo potestatis subsumiert werden können. Für Clemens VI. stellte das Konzept der plenitudo potestatis eine conditio sine qua non für das Amt des Pontifex dar. Er sah sich als Fels, auf dem auch die Kardinäle gegründet seien ${ }^{8}$. Die konkreten Auswirkungen päpstlicher Vollgewalt wurden von ihm in der anläßlich der Bestätigung Karls IV. als römisch-deutscher Kaiser gehaltenen Ansprache eindrucksvoll artikuliert. Päpstliche eminentia und auctoritas erscheinen als Ausfluß dreier exklusiv dem Papst vorbehaltener Funktionen: approbare, iudicare, regulare ${ }^{9}$. Selbst die Stellung der legati a latere, die »aus der Seite « des Papstes heraus entsandt gleichsam als pars corporis pape handeln, wurde von ihm nicht in dem Sinne interpretiert, daß Kardinallegaten von den ihnen übertragenen Vollmachten selbständig Gebrauch machen könnten, ihnen also ein Freibrief für eigenes Handeln ausgestellt worden wäre. Die päpstliche Vollgewalt war unteilbar und bedurfte der Rückkoppelung an die Person des Papstes - nobis inconsultis sollten keine rechtlich bindenden Schritte unternommen werden ${ }^{10}$. Die legatorischen Vollmachten waren also relativ und bedurften zu ihrer vollständigen Realisierung eines päpstlichen Placet.

Unstrittig blieb jedoch trotz aller Relativierung die Stellung des Kardinalskollegiums, dessen Mitglieder als cardines terre, als gigantes agierten, deren

5 Vgl. Collationes Egrediebatur et intrabat, Homo quidam nobilis, Kap. 5.2.2.2., 5.2.4.3.

6 Vgl. Collationes Egrediebatur et intrabat, Kap. 5.2.2.2.; Sicut frigus nivis, Kap. 5.2.1.2.; Hii sunt viri, Kap. 5.1.9.

7 Auch die vom Papst bemühten Namensetymologien sind in diesem Zusammenhang wenig aussagekräftig, vgl. Collationes Ibant et revertebantur, Hii sunt viri, Kap. 5.1.4., 5.1.9. Der unpersönliche, die Grenzen der Gemeinplätze selten überschreitende Charakter der Predigten ermöglichte deren Wiederverwendung auf dem Konzil von Konstanz rund 70 Jahre später, vgl. WooD, Sermon literature, S. 168-172.

8 Vgl. Collatio Egrediebatur et intrabat, Kap. 5.2.2.2.

9 Vgl. Collatio Salomon sedebit super solium meum, in: MGH, Constitutiones, Bd.8, S. 143-162, hier S. 153f.: Papa habet actum primum preceptivum, qui est approbare; papa habet actum preceptivum, quia iudicare, papa habet actum preceptivum, quia regulare. Quia ergo istos tres actus preceptivos habet, idcirco apparet eius eminentia et auctoritas [...]. Preceptum apostolicum preceptum Dei est [...]. Und der Papst schlußfolgert: Potestas enim imperialis catholica et approbata a papa originatur, a papa exemplatur, ad papam terminatur. 10 Vgl. Kap. 5.2.4.1. 
Einflußbereich die gesamte Welt umfaßte. Als colonnae Ecclesie stützten sie das Gebäude der Kirche, waren somit auch Stützen des Papstes, halfen seiner Verzagtheit und Schwachheit auf ${ }^{11}$. In seinem Hang zur Anschaulichkeit ging Clemens VI. noch einen Schritt weiter: das Kardinalskolleg wurde mit dem Himmel verglichen und auffällig nahe an die himmlische Gottesstadt gerückt ${ }^{12}$. $\mathrm{Da}$ die besondere Stellung des Kardinalats eine Überlegenheit gegenüber Bischöfen und Erzbischöfen implizierte, wurde von ihm ebenso wie die Qualifikationen, über die die Mitglieder des Kollegs verfügen sollten, ausführlich dargelegt ${ }^{13}$. Allein die Erhebung des selbst für avignonesische Verhältnisse ausgesprochen jungen Nepoten Pierre Roger de Beaufort veranlaßte Clemens VI. zu dem Eingeständnis, daß auch im Falle einer bereits vorhandenen Sachkompetenz innerhalb des Kollegiums die Erhebung eines zwar jungen, deshalb jedoch nicht unbedingt unreifen Purpurträgers legitim sei. Von den in den Kardinalsstand erhobenen Neffen, die der Papst unter dem Begriff facturae et creaturae subsumierte, verlangte er vor allem eines: bedingungslosen Gehorsam gegenüber dem vicarius Christi. Diese Art des Gehorsams wußte der Papst durch verwandtschaftliche Bande zusätzlich gefestigt ${ }^{14}$. Als Schlüsselqualifikationen, die für eine Kreation vorausgesetzt wurden, galten Reinheit, Weisheit, Redegabe, Strenge, Wohltätigkeit und Gehorsam gegenüber dem Papst ${ }^{15}$. Dies sollte die Ausübung insbesondere der judikativen Tätigkeiten - umschrieben mit dem Begriff causae arduae - garantieren ${ }^{16}$. Des weiteren wurde von Neuzugängen ein Zurücknehmen der eigenen Person mit dem Ziel einer besseren Eingliederung in das Kardinalskollegium erwartet. Clemens VI. unterstrich, daß wichtiger als alle der Zugehörigkeit zu unterschiedlichen kardinalizischen ordines geschuldete Präzedenz die Eintracht sei: Nur caritate coniuncti sei ein fruchtbares Wirken möglich ${ }^{17}$. Mit Parteiungen innerhalb des Kollegs war er in der Tat nicht nur vor dem Hintergrund der Wirren um das Königreich Neapel konfrontiert ${ }^{18}$.

Die Gefahren, denen sich Kardinäle ausgesetzt sahen, wurden hauptsächlich indirekt artikuliert. Unter Bezugnahme auf Gregor den Großen wurde ein ursprünglich den Bischöfen zugewiesenes, auf Gier, Ausschweifung, Dummheit und Neid beruhendes Fehlverhalten benannt und um zwei weitere,

11 Vgl. Collatio Loquere tu et audiemus, Kap. 4.4.

12 In einem gewagten Avignon-Jerusalem-Vergleich verdeutlicht der Papst seine Auffassung, die Nuntien kehrten nach vollendeter Tätigkeit nach Avignon zurück, das als Sitz der Kurie eine Mittelstellung zwischen irdischem und himmlischem Jerusalem einnehme, vgl. Kap. 5.1.9.; vgl. auch Collatio Videntibus illis, Kap. 4.6.

$13 \mathrm{Vgl}$. Collatio Loquere tu et audiemus, Kap. 4.4.

14 Vgl. Collationes Obsecro vos, Kap. 4.2.; Assumpsi michi duas virgas, Kap. 4.3.; Loquere tu, Kap. 4.4.

15 Vgl. Collatio Super montem, Kap. 4.7.

16 Vgl. Collatio Loquere tu et audiemus, Kap. 4.4.

17 Vgl. Collatio Fecit in domo, Kap. 4.5.

18 Verwiesen sei hier nur auf die Feindschaft zwischen den beiden herausragenden Persönlichkeiten des Kollegs, Élie Talleyrand de Périgord und Gui de Boulogne, vgl. Kap. 5.2.4.2. 
diesmal direkt auf die Kardinäle bezogene gravamina ergänzt. Als große Gefahren galten dem Papst die allzu große prosperitas und die Versuche der Einflußnahme von außen. Angesprochen waren also der in einigen Fällen exorbitante Reichtum der Purpurträger - zu dem nicht zuletzt Clemens VI. selbst durch eine exzessive Pfründenvergabe beigetragen hatte ${ }^{19}$ - und das Agieren im Interesse ausländischer Mächte ${ }^{20}$.

In der Vorstellung des Papstes bedurften sämtliche Handlungen der Kardinäle gewisser moventia, deren alleiniger Ursprung in ihm selbst lag. Die Kardinäle waren ihm als dem dominus terre klar untergeordnet und kehrten nach vollendeter Legationstätigkeit vor den Thron Gottes, des Vaters, der in kühner Gleichsetzung zum Thron des Papstes wurde, zurück ${ }^{21}$. Diese unbescheidene Vergleichsebene wurde noch weiter bemüht: Nuntien und Legaten fungierten als viri electi, als »erwähltes Volk «, dessen eminente Stellung auf Gott selbst, stärker aber noch auf den Papst zurückzuführen sei. Dabei wurde der Rangunterschied zwischen den legatorischen Klassen der nuntii, legati und legati a latere durch die Einführung einer kirchenrechtlich nicht nachweisbaren Position eines Nuntius a latere minimiert ${ }^{22}$.

Es dürfte deutlich geworden sein, daß sich Clemens VI. keinesfalls »willfährig « ${ }^{23}$ gegenüber seinen Kardinälen zeigte. Er war sich der Bedeutung von Geben und Nehmen und des labilen Gleichgewichts zwischen Leistung und Remuneration bewußt. Gleichwohl war das Gleichgewicht zwischen seinen autokratischen Vorstellungen und den oligarchischen Bestrebungen seiner Kardinäle Belastungsproben ausgesetzt. Vor diesem Hintergrund verdient festgehalten zu werden, da $\beta$, spricht man von »den Kardinälen " unter Clemens VI., damit zwar die Gesamtheit des Kardinalskollegs gemeint ist, die Bestrebungen dieses Kollegs jedoch nur mit Blick auf einzelne, herausragende Persönlichkeiten deutlich werden. Ein großer Teil der von Clemens VI. zu Kardinälen erhobenen Kleriker trotzt allen Bemühungen um eine exakte Bestimmung ihrer Macht. Ein Blick auf die im Appendix aufgeführten Kardinalsbiogramme verdeutlicht, daß viele Kardinäle nichts anderes als ein Leben im Hintergrund führten - ein Leben, das von Clemens VI. freilich durch die Zuweisung zahlreicher Pfründen abgesichert wurde. Oligarchische Bestrebungen sind während des Pontifikates Clemens' VI. nur schwer nachweisbar und verblassen gleichsam vor dem Hintergrund rhetorischer Machtdemonstrationen des Papstes. Daß einzelne Kardinäle das Ungleichgewicht zwischen ihrer eigenen Position und der des Papstes als drückend empfanden, belegt der von Kardinal Bertrand du Pouget gehaltene Sermo Erat Jhesus eiciens, in

$19 \mathrm{Vgl}$. hier die in den einzelnen Biogrammen vorgenommene Auflistung der von Clemens VI. vergebenen Pfründen.

$20 \mathrm{Vgl}$. Collatio Fecit in domo, Kap. 4.5.

21 Vgl. Kap. 5.1.9.

22 Vgl. ibid.

${ }^{23}$ LULVĖs, Machtbestrebungen, S. 101. 
dem vorsichtig formuliert auf die Stellung der Kardinäle als Apostelnachfolger eingegangen wurde ${ }^{24}$. Clemens selbst hatte Stellungnahmen zu diesem heiklen Thema tunlichst vermieden und lediglich an einer einzigen Stelle seines Predigtcorpus den Kardinälen den Rang von successores Apostolorum zugebilligt ${ }^{25}$. Der von der Forschung immer wieder bemühte Verweis auf die 1352 im Konklave verabschiedete Wahlkapitulation, von vielen als erste Verschriftlichung der unter den avignonesischen Päpsten, insbesondere unter Clemens VI., stark angewachsenen Teilhabe der Kardinäle an der Macht, greift meiner Ansicht nach entschieden zu kurz.

Der Papst entwickelte zwar keine eigenständige Theologie des Kardinalats, artikulierte seine Vorstellungen von dessen Bedeutung jedoch an keiner Stelle deutlicher als in den anläßlich seiner Kardinalskreationen gehaltenen Predigten. Er verzichtete in ihnen auf die Darstellung einer von gleichberechtigtem Miteinander geprägten Kollegialität ${ }^{26}$. Zwar versicherte er die Kardinäle einer Teilhabe an der päpstlichen plenitudo potestatis, versäumte es jedoch nicht, sogleich die Grenzen dieser Partizipation zu markieren - Non solum assumuntur ad partem sollicitudinis, sed quodammodo in plenitudinem potestatis ${ }^{27}$. Kardinäle wurden von ihm als Teil des mystischen Papstleibes begriffen, was jedoch nicht von einer Unter- zu einer gleichberechtigten Nebenordnung führte. Clemens VI. erwartete von seinen Kardinälen nicht Unterwürfigkeit, sondern Loyalität und damit ein Verhalten, das seine eigenen Handlungen maßgeblich mitbestimmte. Diese päpstliche Loyalität manifestierte sich immer dann, wenn Kardinäle zur Zielscheibe verleumderischer Angriffe wurden. Egal, ob entsprechende Vorwürfe von weltlichen Souveränen an den Papst adressiert wurden oder ihren Ursprung an der Kurie hatten: in den meisten Fällen verwahrte er sich energisch dagegen, wohl wissend, daß Angriffe gegen die Kardinäle sich auch gegen ihn selbst richten konnten ${ }^{28}$. Daher stellte er sich auf

24 Vgl. Kap. 5.1.10.

25 Vgl. Collatio Videntibus illis, Kap. 4.6. Dieser Bezug resultiert aus den thematischen Vorgaben: so wie die Apostel der Himmelfahrt Christi staunend beigewohnt hätten, seien nun die Kardinäle gleichsam Zeugen der Himmelfahrt des Pierre Roger de Beaufort, d.h. seiner Aufnahme ins Kolleg. Die Gleichsetzung erfolgt somit als kaum zu vermeidender Analogieschluß.

26 Vgl. Yves Congar, Notes sur le destin de l'idée de collégialité épiscopale en Occident au Moyen Âge (VII - XVI ${ }^{\mathrm{e}}$ siècles), in: La collégialité épiscopale. Histoire et théologie, Paris 1965, S. 99-131, bes. S.127: "Enfin, avec la croissance du rôle des cardinaux, de très bons esprits ont été, pendant près de deux siècles, trompés par la chimère d'une institution divine des cardinaux: l'idée de succession apostolique et celle de collégialité ont été ainsi largement confisquées par l'idéologie d'une fonction cardinalice à laquelle, par surcroît, on attribuait ce que personne n'a jamais réclamé pour la collégialité épiscopale, à savoir le partage de la fonction même du pape et de sa primauté«. Diese Überlegungen sind in ihrer Algemeinheit auf die Situation unter Clemens VI. nur bedingt anwendbar; vgl. zum Kollegialitätsbegriff auch ALBERIGo, Cardinalato e collegialità.

27 MS 240, fol. 416va; Kap. 4.6.

28 Vgl. Kap. 5.2.3.2. 
die Seite von Étienne Aubert und Annibaldo Ceccano, als ihnen von der französischen Königin die Schuld an der Katastrophe von Crécy zugeschrieben wurde ${ }^{29}$. Für seine Nuntien fürchtete er zu diesem Zeitpunkt nicht nur um den durch die Verleumdungskampagne unterminierten Ruf, sondern ebenso um ihre körperliche Unversehrtheit. Gleichwohl wurde eine Rückkehr an die Kurie mit dem Argument untersagt, der honor Ecclesie könne auch den Einsatz des Lebens notwendig werden lassen ${ }^{30}$. Im Falle des Annibaldo Ceccano sollte sich dieser Grundsatz bewahrheiten: In Rom entkam dieser nur knapp einem Attentat $^{31}$. Guillaume Court, der sich während seiner Legation nach Oberitalien mit dem Vorwurf der Bestechlichkeit und Vorteilsnahme im Amt konfrontiert sah, profitierte ebenfalls von der Loyalität des Papstes, der im Angriff auf ihn auch eine gegen sich selbst gerichtete Kampagne antipäpstlicher Kreise $\operatorname{sah}^{32}$. Das gilt auch für Bertrand de Deux, dessen energisches Vorgehen gegen Cola di Rienzo in Rom zunächst nicht ungeteilte Zustimmung fand und eine vom Papst unpräzise als aliqua nobis contra te relata et scripta umschriebene Intervention zur Folge hatte. Doch auch in diesem Fall stellte sich der Papst schützend vor seinen Legaten ${ }^{33}$. Er verwahrte sich ebenfalls energisch gegen Anschuldigungen, die sich nach dem gewaltsamen Tod Andreas' von Ungarn gegen das gesamte Kolleg richteten ${ }^{34}$.

An der Kurie selbst artikulierte sich die Unzufriedenheit mit dem Vorgehen einzelner Legaten zumeist nicht in Form anonymer Verleumdungen, sondern in Gestalt veritabler, dem Papst vorgetragener Beschwerden. Aimeric de Châtelus wurde z. B. mangelnder Verhandlungswille in Fragen der von Neapel $\mathrm{zu}$ leistenden Census-Zahlungen vorgeworfen ${ }^{35}$, Bertrand de Deux wurde Opfer von Angriffen, die sich gegen sein selbstherrliches Kollationsgebaren richteten ${ }^{36}$. In beiden Fällen gingen die Anklagen auf die Mitglieder des Kardinalskollegs selbst zurück, die sich durch das Vorgehen der Legaten in ihren finanziellen Interessen beschnitten sahen. Die Reaktionen des Papstes fielen unterschiedlich aus: Der seit langem unglücklich agierende Aimeric de Châtelus wurde gerügt, während Bertrand de Deux aufgefordert wurde, zukünftig die berechtigten Interessen der übrigen Kardinäle bei seinen Handlungen zu berücksichtigen.

${ }^{29}$ Vgl. Kap. 5.1.8.; vgl. auch MS 240, fol.451ra. In der Collatio spricht der Papst vom labium detractorum und verweist diese Art von Verleumdung in den Bereich der von den Nuntien durchlittenen tribulationes.

30 Im Falle des Bertrand de Deux zeigte sich der Papst konzilianter. Als die Situation in Neapel zu eskalieren drohte, erging die Weisung, sich auf außerneapolitanisches Territorium zu begeben, vgl. Kap. 5.2.3.1. Honor Ecclesie und honor cardinalium waren hinsichtlich der Glaubwürdigkeit päpstlicher Diplomatie identisch, vgl. Kap. 5.2.3.2.

31 Vgl. Kap. 5.2.4.1.

32 Vgl. Kap. 5.2.1.1.

33 Kap. 5.2.3.1.

34 IBID.

35 Kap. 5.2.2.3.

36 Kap. 5.2.3.1. 
Clemens VI. zögerte nicht, sein Prestige in Verhandlungen einzubringen, deren Erfolg alles andere als garantiert war. Daß er trotz eines immer möglichen Scheiterns, das seine Macht und sein Ansehen hätte unterminieren können, nicht zögerte, Europa mit einem dichten Netz von Gesandten in Kardinalsrang zu überziehen, spricht für seinen verantwortungsvollen Umgang mit der ihm zustehenden plenitudo potestatis. Anders als im Falle der Kreationen war seine Personalpolitik, was die Legationen angeht, maßgeblich von seinen eigenen Erfahrungen bestimmt. Im Konflikt zwischen England und Frankreich wollte er nicht nur Notar eines möglichen Friedensschlusses sein, wollte nicht nur beglaubigen und garantieren, worauf andere sich geeinigt hatten, sondern aktiv und an prominenter Stelle an der Friedensstiftung beteiligt sein. Sieht man von dem Intermezzo der Konferenz von Avignon $1344 \mathrm{ab}$, an der Clemens VI. in persona beteiligt war, oblagen die Verhandlungen den jeweils entsandten Kardinälen, die in enger Rückbindung an die Kurie nicht nur über einen Waffenstillstand, sondern auch über weitergehende politische Lösungen verhandeln sollten. Friedensverhandlungen konnten sie ebenso wie der Papst jedoch nur so weit vorantreiben, wie es die Konfliktparteien zuließen, was das Scheitern fast aller Legationen z. T. erklären kann. Der Begriff des Scheiterns bedarf der Relativierung, wurden von den Diplomaten des Heiligen Stuhls doch auch positive, obgleich ephemere Ergebnisse in Form von Waffenstillstandsabkommen erzielt ${ }^{37}$. So konnte der im Königreich Neapel glücklos agierende Annibaldo Ceccano mit der gelungenen Organisation des Heiligen Jahres 1350 in Rom und der Vermittlung eines Waffenstillstands in der Romagna durchaus auch Erfolge verbuchen $^{38}$.

Im Falle der als Legaten amtierenden Kardinäle, bei denen eine Verschränkung geistig-moralischer und praktischer Qualitäten vorausgesetzt wurde $^{39}$, gemahnt deren Agieren in vielen Punkten an das, was Max Weber

37 Im Falle der Abkommen von Malestroit (1343) und Calais (1347) wurden diese zwar als Frucht kardinalizischer Vermittlungstätigkeit gepriesen, waren jedoch weniger auf das geschickte Taktieren der Kardinäle vor Ort, denn auf den Willen Edwards III., eine Kriegspause einzulegen, zurückzuführen.

38 Kap. 5.2.4.1.

39 Vgl. Collatio Legatus fidelis sanitas, Kap. 5.2.3.2. Als Eigenschaften der Kardinallegaten, die fortes opere et sermone operieren sollten, wurden konkret benannt: Bildung, Fleiß, Reinheit, Unbestechlichkeit, Treue, Demut, Stärke, Sanftmut, Klarheit, Weitsicht, insgesamt ein Bemühen um Ausgleich, Gerechtigkeit und Friede, vgl. Kap. 5.2.1.2., 5.2.2.3. Alle erwähnten Gesichtspunkte fanden bereits in der Einleitung der Collatio Et iste bonus est nuntius, gehalten am 10. September 1343 anläßlich der Rückkehr von Kardinal Bernard d'Albi, Erwähnung, vgl. MS 240, fol. 68ra : Videtur michi, quod bonitas solempnis nuncii in quatuor spiritualiter colligitur et apparet. Primo in intelligendo et recolligendo mandata prudenter et subtiliter; secundo in prosequendo comissa diligenter et viriliter; tercio in consummando iniuncta salubriter et utiliter; quarto in referendo audita et gesta fideliter et veraciter. Bonus enim nuntius dici potest, qui est capax et sagax in intelligendo et recolligendo, qui est vivax et audax in prosequendo et continuando, qui est verax in significando et referendo, qui est efficax in concludendo et consummando. 
1919 als Charakteristikum des Politikers bezeichnete: Leidenschaft, Verantwortungsgefühl und Augenma $\beta^{40}$. $\mathrm{Da}$ die diplomatische Arbeit vor Ort jedoch anderen Gesetzmäßigkeiten folgte, idealtypisches Agieren erschwerte, wenn nicht gar unmöglich machte, zeigt die Intervention der Kurie im Königreich Neapel. Das Scheitern aller drei in diese Region entsandten Legationen war nicht allein den unmittelbar involvierten dramatis personae, also den Vertretern der Verhandlungsparteien, anzulasten. Die Kardinallegaten agierten zwar nicht selten ungeschickt und hilflos, waren jedoch auch einer Obstruktionspolitik ausgesetzt, die den Einsatz ihrer umfangreichen Fakultäten nutzlos werden ließ. Dazu gesellte sich eine Form von Obstruktion, deren Quelle die Kurie selbst war. Seiner Vorstellung folgend, jedwede moventia der Kardinäle persönlich zu beeinflussen, forderte Clemens VI. eine enge Rückkoppelung der Legaten an seine Person. Daß damit unmittelbares und zielgerichtetes Agieren erschwert, ja oft unmöglich gemacht wurde, versteht sich von selbst. Auch die vom Papst häufig betriebene Doppeldiplomatie war nicht dazu angetan, die Stellung und das Ansehen der Kardinallegaten zu erhöhen. Für einen vermeintlichen Informationszuwachs nahm der Papst also die Schwächung der Position seiner Legaten in Kauf. Die in der kurialen Korrespondenz artikulierten Vorwürfe von pigritia und inertia ${ }^{41}$ waren daher oft ungerecht, weil direkte Konsequenz der päpstlichen Strategie. Die Entscheidungskompetenz behielt sich der Papst in allen Dingen vor. Obgleich für ihn der Kardinalat erst in Verbindung mit einer Legation zur vollen Entfaltung gebracht wurde ${ }^{42}$, wird die Resignation verständlich, die einige Legaten ihre Abberufung herbeisehnen lie $\beta^{43}$.

Auch noch für das 14. Jahrhundert läßt sich konstatieren, daß Einfluß und Mitsprache der Kardinäle an der päpstlichen Universalmonarchie »nicht von einer geregelten Übertragung von Kompetenzen, sondern vorwiegend von der Stärke oder Schwäche der Persönlichkeiten auf dem Stuhl Petri oder im Kardinalskollegium abhingen “ $^{44}$. Clemens VI. agierte als starker Papst und wußte die auf Unabhängigkeit und stärkere Teilhabe an der Macht zielenden Bestrebungen der Kardinäle wirkungsvoll zu beschränken. Vor diesem Hintergrund sollte die im folgenden Konklave 1352 von den Purpurträgern beschworene Wahlkapitulation dazu beitragen, sich auf der Grundlage rechtlich bindender Verfügungen aus dieser Beschränkung zu befreien.

40 Max Weber, Politik als Beruf, München 1919, S. 3.

$41 \mathrm{Zu}$ diesen wenig schmeichelhaften Worten griff Clemens VI. im Falle des Bertrand de Deux, vgl. Kap. 5.2.3.1.

$42 \mathrm{Vgl}$. Collatio Egrediebatur et intrabat, Kap. 5.2.2.2. Mit der innerhalb der Collatio Homo quidam nobilis getätigten Gleichsetzung des officium legationis mit dem regnum wurde das Legatenamt gar in die Nähe der nach Aristoteles bestmöglichen Herrschaftsform gerückt, vgl. Kap. 5.2.4.3.

43 Das beste Beispiel liefert hier Guillaume Court, vgl. Kap. 5.2.1.1.

44 MALECZEK, Papst und Kardinalskolleg, S. 207f. 
Die Aussage, zu der sich Calixt II. zu Beginn des 12. Jahrhunderts hinreißen ließ - quando vero Rome ero, quot cardinales, tot principes, tot domini erunt $t^{45}$, galt für seine in Avignon residierenden Nachfolger nur bedingt. Clemens VI. hatte sich im Laufe seines Pontifikates an die Mahnung der Birgitta von Schweden gehalten. Geschick bei der Auswahl von Kandidaten für die Kardinalswürde trug im Verbund mit einem System von Geben und Nehmen dazu bei, seine Stellung als Summus Pontifex zu festigen und den oligarchischen Bestrebungen der Kardinäle wirkungsvoll entgegenzutreten. Birgitta von Schweden konnte zufrieden sein. Ihr Wunsch war in Erfüllung gegangen: Flexit cardinales ad velle suum.

45 Vgl. Hugo CanToR, The History of the Church of York 1066-1127, hg. und übersetzt von Charles JohNSON, überarbeitet von Martin BrETT, Cecile Brooke, Michael WinTERвоттом, Oxford 1990, S.55. 
